\title{
Unique identification of phonon modes using polarized Raman studies of $\mathrm{SnO}(001)$ crystals
}

\author{
RAKTIMA BASU ${ }^{1}$, D SORNADURAI ${ }^{2}, S_{\text {AMIRTHAPANDIAN }}^{3}$ and SANDIP DHARA ${ }^{1, *}$ (D) \\ ${ }^{1}$ Surface and Nanoscience Division, Indira Gandhi Centre for Atomic Research, Homi Bhabha National Institute, \\ Kalpakkam 603102, India \\ ${ }^{2}$ Condensed Matter Physics Division, Indira Gandhi Centre for Atomic Research, Kalpakkam 603102, India \\ ${ }^{3}$ Materials Physics Division, Indira Gandhi Centre for Atomic Research, Homi Bhabha National Institute, \\ Kalpakkam 603102, India \\ *Author for correspondence (dhara@igcar.gov.in)
}

MS received 9 June 2018; accepted 9 August 2018; published online 15 March 2019

\begin{abstract}
Stannous oxide ( $\mathrm{SnO}$ ), an exclusive p-type oxide semiconductor in the oxide family, is a source of renewed interest because of its ability to be an excellent anode material. So far, there are very few reports on the vibrational properties of $\mathrm{SnO}$ and controversy remains in the assignment of vibrational modes. Textured single crystals of SnO were synthesized by a one-step solvothermal method. The as-synthesized $\mathrm{SnO}$ crystals have a wide (001) plane, as confirmed by high resolution transmission electron microscopy images. Raman spectroscopy is used for the identification of phase as well as crystalline orientation. Moreover, a unique assignment of phonon modes in $\mathrm{SnO}$ is also performed using polarized Raman spectroscopic studies for different orientations around $c$-axis of the crystal with the incident electric field vector. Thus, a novel methodology of phonon assignment is adopted with a minimum amount of data collection for a diatomic molecule having a tetragonal symmetry with a number of symmetry elements.
\end{abstract}

Keywords. Polarized Raman spectroscopy; SnO; Raman mode; solvothermal technique; crystal orientation.

\section{Introduction}

Stannous oxide $(\mathrm{SnO})$ finds significant applications in thin film transistors [1], coating substances, as a catalyst for the polymerization of lactic acids [2-4] and as a precursor for the production of $\mathrm{SnO}_{2}$, which is an important electronic grade material for sensor applications [5]. SnO is a p-type semiconductor with a direct optical band gap in the range of $2.7-3.4 \mathrm{eV}$ and is recently found to be a promising anode material for lithium-ion rechargeable batteries [6,7]. It is well known that the optical properties of the material are determined by its structural symmetry. As the properties of metal oxides depend on unique structures and morphologies, various $\mathrm{SnO}$ structures such as particles, meshes, sheets, belts and blocks are being synthesized by a variety of methods $[6,8-11]$. There are very few reports on the vibrational properties of $\mathrm{SnO}[12,13]$. Two Raman peaks at 114 and $211 \mathrm{~cm}^{-1}$ are reported for $\mathrm{SnO}$ $[14,15]$. However, controversy remains in the assignment of the peaks both in theoretical calculations and experimental reports [15-19]. Although the Raman peak at $210 \mathrm{~cm}^{-1}$ is identified as $A_{1 \mathrm{~g}}$ mode, there is a conflict in the assignment of the peak centred at $110 \mathrm{~cm}^{-1}$. The peak is assigned as $E_{\mathrm{g}}$ mode as well as $B_{1 \mathrm{~g}}$ mode in the experimental reports of a polycrystalline sample $[15,16]$. However, for a randomly oriented polycrystalline structure, the assignment of Raman modes may be obscure at times, as the net intensity may lead to zero or get reduced for two oppositely oriented crystallites. On the other hand, low-frequency $E_{\mathrm{g}}$ mode ranges from 113 to $143 \mathrm{~cm}^{-1}$, while $B_{1 \mathrm{~g}}$ mode is speculated between 350 and $370 \mathrm{~cm}^{-1}$ according to theoretical calculations $[18,19]$. Thus, a systematic polarized Raman study on oriented $\mathrm{SnO}$ single crystal is required for the definite assignment of the mode frequencies.

In the present study, we have reported detailed structural and vibrational properties of $\mathrm{SnO}(001)$ single crystals. Raman spectroscopy is used as an easy tool for the identification of phase as well as crystalline orientation. Polarized Raman spectroscopic analysis is used for the identification of different phonon modes in tetragonal $\mathrm{SnO}$.

\section{Experimental}

$\mathrm{SnO}$ crystals were synthesized in a hydrothermal process using $\mathrm{SnCl}_{2}(10 \mathrm{mM})$ dissolved in equal volume of dimethyl formamide and water solvent ( $40 \mathrm{ml}$ of each) in a Teflon lined stainless steel autoclave. The reaction was carried out at $140^{\circ} \mathrm{C}$ for $72 \mathrm{~h}$. The auto-cooled solution was filtered and washed with distilled water and dried at $60^{\circ} \mathrm{C}$ for $30 \mathrm{~min}$ yielding bluish black coloured iridescent cubicand cuboidal-shaped crystals. Compositional stability and detailed synthesis process are reported elsewhere [20]. 
Morphological analysis of the pristine sample was studied using a field emission scanning electron microscope (FESEM, SUPRA 55 Zeiss). The structural and crystallographic nature of the $\mathrm{SnO}$ crystals was investigated using high resolution transmission electron microscopy (HRTEM, LIBRA 200FE Zeiss). The TEM sample was prepared by dispersing in a $\mathrm{Cu}$ grid by using isopropyl alcohol. The vibrational modes of the pristine sample were studied by Raman spectroscopic analysis using a micro-Raman spectrometer (inVia, Renishaw, $\mathrm{UK})$ in the backscattering configuration with an $\mathrm{Ar}^{+}$laser $(514.5 \mathrm{~nm})$ as the excitation source, diffraction gratings of $1800 \mathrm{~g} \mathrm{~mm}^{-1}$ as a monochromator and a thermoelectrically cooled CCD camera as the detector. The spectra were collected using $50 \times$ objective with a numerical aperture of 0.35 . To perform polarized Raman studies, half-wave plates and polarizer were inserted accordingly in the scattered ray path to achieve the required configurations.

\section{Results and discussion}

The tetragonal structure of $\mathrm{SnO}$ belongs to the $P 4 / n m m\left(D_{4 \mathrm{~h}}\right)$ space group. The schematic structure (figure 1a) is layered with an AAK stacking sequence of slabs consisting of $\mathrm{O}$ ions sandwiched between $\mathrm{Sn}$ ions. The $\mathrm{Sn}$ ions form four square pyramidal bonds with Os [13]. The Cartesian $X, Y$ and $Z$ axes are chosen along $a, b$ and $c$ directions of the crystal, respectively (figure 1a). Detailed structural studies are reported earlier [20].

FESEM image (figure 1b) of the grown sample shows a typical single microcrystal of dimension $\sim 300 \times 100 \mu \mathrm{m}$. The inset of figure $1 \mathrm{~b}$ shows the corresponding optical image of the microcrystal. HRTEM images were acquired for the pristine $\mathrm{SnO}$ crystals to identify the crystallographic orientation of the samples used for optical measurements. Figure 2 shows HRTEM lattice images and the corresponding fast Fourier transform (FFT) pattern of the $\mathrm{SnO}$ crystal.

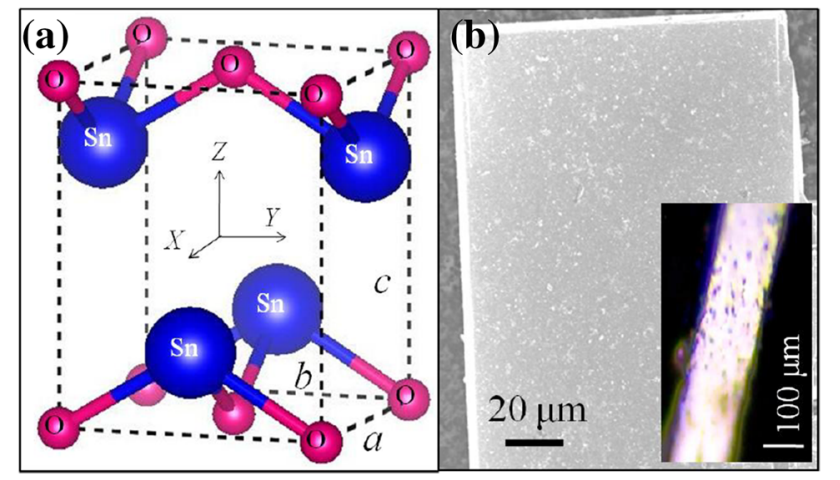

Figure 1. (a) Schematic structural diagram of $\mathrm{SnO}$ unit cell. The Cartesian $X, Y$ and $Z$ axes are chosen along $a, b$ and $c$ directions of the crystal, respectively. (b) FESEM image of the as-grown microcrystal. The inset shows corresponding optical image of the crystal.

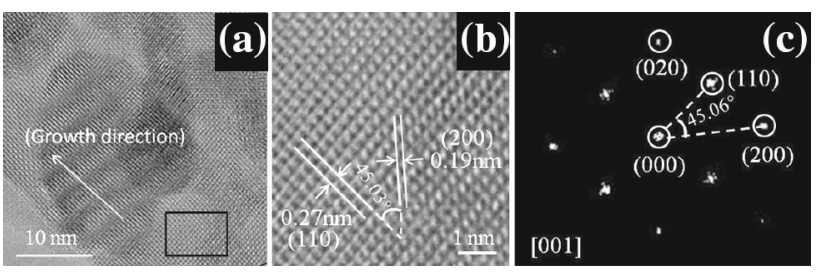

Figure 2. (a) HRTEM images of the as-synthesized $\mathrm{SnO}$ crystal. Arrow indicates the growth direction. (b) Enlarged image of the selected area from (a) showing $(h k l)$ planes and (c) the corresponding FFT pattern of tetragonal phase of $\mathrm{SnO}$ with zone axes [001].

In the HRTEM image (figure 2a) and the magnified TEM image (figure $2 \mathrm{~b}$ ), the inter-planar spacing $(d)$ values are found to be 0.27 and $0.19 \mathrm{~nm}$, which match with the reported $d$ values for the (110) and (200) planes of SnO, respectively (JCPDS card no. 06-0395). The corresponding FFT pattern of the $\mathrm{SnO}$ crystal (figure 2c) is indexed to the tetragonal phase of single-crystal $\mathrm{SnO}$ with zone axes along [001]. In both HRTEM image and FFT pattern, the angle between the planes is $45^{\circ}$. The TEM analysis confirms that the growth direction of the pristine $\mathrm{SnO}$ crystal (long axis) is along [110] and the crystal has a single-crystal-like structure with a wide, well-ordered (001) plane [21,22].

Raman spectroscopy is used to understand the vibrational properties of a material while interacting with electromagnetic excitation. The electric field vectors of the Raman scattered wave is associated with the electric field vector of the incident wave with a characteristic Raman tensor. There exists a unique Raman tensor for each Raman-active vibrational mode. The electric field vectors of the incident radiation $\left(\boldsymbol{E}_{x \mathrm{i}} ; \boldsymbol{E}_{y \mathrm{i}} ; \boldsymbol{E}_{z \mathrm{i}}\right)$ relates with the electric field vectors of the Raman scattered radiation $\left(\boldsymbol{E}_{x \mathrm{~s}} ; \boldsymbol{E}_{y \mathrm{~s}} ; \boldsymbol{E}_{z \mathrm{~s}}\right)$ by the Raman tensor $(R)$ as shown below:

$$
\left(\begin{array}{c}
E_{x \mathrm{~s}} \\
E_{y \mathrm{~s}} \\
E_{z \mathrm{~s}}
\end{array}\right)=\left(\begin{array}{lll}
R_{x x} & R_{x y} & R_{x z} \\
R_{y x} & R_{y y} & R_{y z} \\
R_{z x} & R_{z y} & R_{z z}
\end{array}\right)\left(\begin{array}{c}
E_{x \mathrm{i}} \\
E_{y \mathrm{i}} \\
E_{z \mathrm{i}}
\end{array}\right) .
$$

Group theoretical analysis predicts four Raman active modes for $\mathrm{SnO}$ at $\Gamma$ point, $A_{1 \mathrm{~g}}+B_{1 \mathrm{~g}}+2 E_{\mathrm{g}}$. The corresponding Raman tensors are shown below [12,23]:

$$
\begin{array}{cl}
A_{1 \mathrm{~g}}:\left(\begin{array}{ccc}
a & 0 & 0 \\
0 & a & 0 \\
0 & 0 & b
\end{array}\right) ; & B_{1 \mathrm{~g}}:\left(\begin{array}{ccc}
c & 0 & 0 \\
0 & -c & 0 \\
0 & 0 & 0
\end{array}\right) ; \\
E_{\mathrm{g}}:\left(\begin{array}{lll}
0 & 0 & 0 \\
0 & 0 & e \\
0 & e & 0
\end{array}\right) ; & E_{\mathrm{g}}:\left(\begin{array}{ccc}
0 & 0 & -e \\
0 & 0 & 0 \\
-e & 0 & 0
\end{array}\right) .
\end{array}
$$

For textured single crystal of $\mathrm{SnO}(001)$, the incident and scattered electric field vectors can have components only 


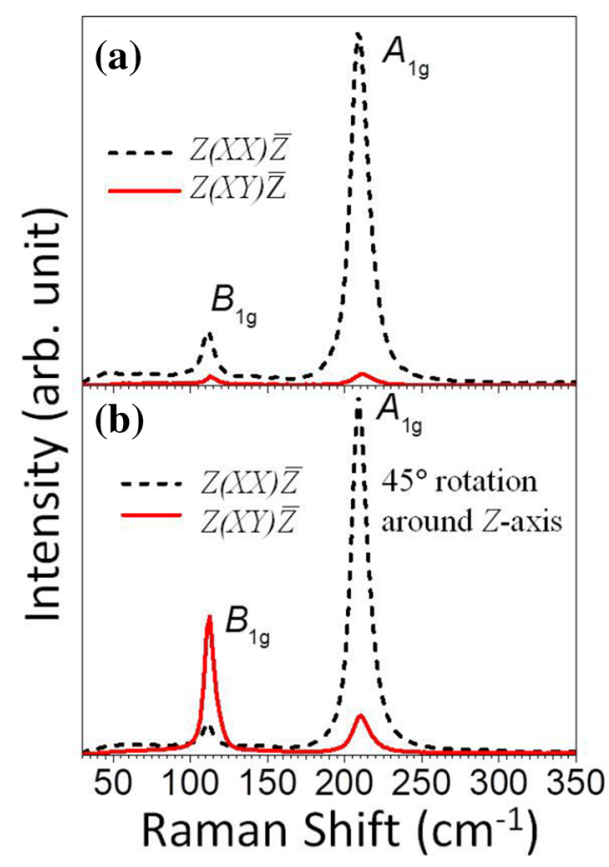

Figure 3. Raman spectra of the (001) textured $\mathrm{SnO}$ crystal at (a) $Z(X X) \bar{Z}, Z(X Y) \bar{Z}$ polarization condition and (b) with a rotation of $45^{\circ}$ around $Z$-axis in $Z(X X) \bar{Z}, Z(X Y) \bar{Z}$ polarization condition.

along $a$ and $b$ crystal axes, as incident and scattered wave vectors are along $Z$ and $\bar{Z}$ axes, respectively. From the above mentioned Raman tensors, it is clear that only $A_{1 \mathrm{~g}}$ and $B_{1 \mathrm{~g}}$ modes are allowed for the (001) textured crystals, as they have non-vanishing $x x$ and $y y$ tensor components. Using equation (1), one can find out both $A_{1 \mathrm{~g}}$ and $B_{1 \mathrm{~g}}$ modes, which are allowed in the $Z(X X) \bar{Z}$ and $Z(Y Y) \bar{Z}$ configurations. Whereas, no mode is allowed in the $Z(X Y) \bar{Z}$ and $Z(Y X) \bar{Z}$ configurations. The first and last letters in the Porto notation denote the incident and scattered wave vectors, respectively, and the letters within the parentheses denote the directions of incident as well as the scattered electric field vectors, respectively. As $\left|R_{i j}\right|=\left|R_{j i}\right|$ ( $i, j$ denote the axes) for all the allowed modes of $\mathrm{SnO}$, we studied the parallel $[Z(X X) \bar{Z}]$ and perpendicular $[Z(X Y) \bar{Z}]$ polarization conditions only.

Raman spectra of the textured $\mathrm{SnO}(001)$ crystals at two different polarization conditions and for two different orientations are shown in figure 3 .

Two Raman modes are observed at 114 and $211 \mathrm{~cm}^{-1}$ in the $Z(X X) \bar{Z}$ configuration [12-14] as expected from our previous discussion. Whereas in the case of $Z(X Y) \bar{Z}$ polarization condition, intensity of both the peaks falls down rapidly (figure 3a). The depolarization ratios $\rho\left(=I_{\perp} / I_{\|}\right.$, where $I_{\perp}$ and $I_{\|}$being the intensities of the Raman modes in the perpendicular $[Z(X Y) \bar{Z}]$ and parallel $[Z(X X) \bar{Z}]$ configurations, respectively) of these two modes are $\rho\left(114 \mathrm{~cm}^{-1}\right)=$ 0.16 and $\rho\left(211 \mathrm{~cm}^{-1}\right)=0.03$. The small values of $\rho$ make the modes highly polarized considering the Placzek's polarizability approximation value of 0.75 . It is also clear that the above two observed modes are $A_{1 \mathrm{~g}}$ and $B_{1 \mathrm{~g}}$, as those are the only allowed modes in the $Z(X X) \bar{Z}$ configuration. However, assignment of the individual mode either for $A_{1 \mathrm{~g}}$ or $B_{1 \mathrm{~g}}$ is not possible from the above study.

For assigning the modes individually, we rotated the crystal by an angle of $45^{\circ}$ around $Z$-axis ( $c$-axis of the crystal) and repeated the polarized Raman experiment. Figure 4 shows the schematic of the generation of scattered modes for two different crystal orientations of $\mathrm{SnO}(001)$ single crystal. In the first case (top panel of figure 4), the electric field vector of the incident wave $\left(\boldsymbol{E}_{\mathrm{i}}\right)$ is parallel to the $X$-axis i.e., $a$-axis of the crystal frame. So, the $x$ and $y$ components of the incident electric field are $\boldsymbol{E}_{x \mathrm{i}}=\boldsymbol{E}_{\mathrm{i}}$ and $\boldsymbol{E}_{y \mathrm{i}}=0$, respectively.

Using equation (1), one can predict the direction of scattered electric field vector for both $A_{1 \mathrm{~g}}$ and $B_{1 \mathrm{~g}}$ modes. For $A_{1 \mathrm{~g}}$ mode, the scattered electric field vectors are $\boldsymbol{E}_{\mathrm{s} x}=a \boldsymbol{E}_{\mathrm{i}}$ and $\boldsymbol{E}_{\mathrm{s} y}=0$. Similarly, for $B_{1 \mathrm{~g}}$ mode, two components are $\boldsymbol{E}_{\mathrm{s} x}=c \boldsymbol{E}_{\mathrm{i}}$ and $\boldsymbol{E}_{\mathrm{s} y}=0$. So, the scattered electric field $\left(E_{\mathrm{s}}\right)$ for both the $A_{1 \mathrm{~g}}$ and $B_{1 \mathrm{~g}}$ modes are along $X$-axis i.e., parallel to $\boldsymbol{E}_{\mathrm{i}}$ and therefore, both the modes are allowed for $Z(X X) \bar{Z}$ configuration only. In the second case (bottom panel of figure 4), the crystal is rotated by an angle $45^{\circ}$ around the $Z$-axis. So, $\boldsymbol{E}_{\mathrm{i}}$ has both $x$ and $y$ components $\boldsymbol{E}_{x \mathrm{i}}$ and $\boldsymbol{E}_{y \mathrm{i}}$ in the crystal frame. Hence, $\boldsymbol{E}_{\mathrm{s}}$ for $A_{1 \mathrm{~g}}$ mode also has two components $\boldsymbol{E}_{\mathrm{s} x}=a \boldsymbol{E}_{x \mathrm{i}}$ and $\boldsymbol{E}_{\mathrm{s} y}=a \boldsymbol{E}_{y \mathrm{i}}$. The resultant $\boldsymbol{E}_{\mathrm{s}}$ is along $\boldsymbol{E}_{\mathrm{i}}$, as for $A_{1 \mathrm{~g}}$ mode, both $x x$ and $y y$ Raman tensor components are equal in magnitude and sign. On the other hand, $x x$ and $y y$ tensor components are equal with opposite sign for $B_{1 \mathrm{~g}}$ mode. Thus, scattered electric field for $B_{1 \mathrm{~g}}$ mode has components, $\boldsymbol{E}_{\mathrm{s} x}=c \boldsymbol{E}_{x \mathrm{i}}$ and $\boldsymbol{E}_{\mathrm{s} y}=-c \boldsymbol{E}_{y \mathrm{i}}$. The total $\boldsymbol{E}_{\mathrm{s}}$ is the vector sum of $\boldsymbol{E}_{\mathrm{sx}}$ and $\boldsymbol{E}_{\mathrm{sy}}$ and is along perpendicular direction of $\boldsymbol{E}_{\mathrm{i}}$. So, $A_{1 \mathrm{~g}}$ mode is allowed only in the parallel $[Z(X X) \bar{Z}]$ polarization configuration, and $B_{1 \mathrm{~g}}$ mode is allowed only in the perpendicular $[Z(X Y) \bar{Z}]$ polarization configuration.

The Raman spectra of the $45^{\circ}$ oriented crystal for different polarization conditions are shown in figure $3 \mathrm{~b}$. In the $Z(X X) \bar{Z}$ configuration, a sharp Raman peak is observed at $211 \mathrm{~cm}^{-1}$. Whereas in the case of $Z(X Y) \bar{Z}$ polarization condition, a sharp peak is observed at $114 \mathrm{~cm}^{-1}$. According to the above discussion, we can assign the Raman modes observed at $211 \mathrm{~cm}^{-1}$ as $A_{1 \mathrm{~g}}$ and $114 \mathrm{~cm}^{-1}$ as $B_{1 \mathrm{~g}}$ uniquely, as $A_{1 \mathrm{~g}}$ and $B_{1 \mathrm{~g}}$ modes are allowed only in the parallel $[Z(X X) \bar{Z}]$ and in the perpendicular $[Z(X Y) \bar{Z}]$ polarization configurations, respectively. Both $A_{1 \mathrm{~g}}$ mode in $Z(X Y) \bar{Z}$ and $B_{1 \mathrm{~g}}$ mode in $Z(X X) \bar{Z}$ conditions are present with negligible intensities, possibly because of polarization leaking considering lack of perfect levelling of the crystal orientations. The $\rho\left(I_{X X} / I_{X Y}\right.$; $\left.114 \mathrm{~cm}^{-1}\right)=0.23$ and $\rho\left(I_{X Y} / I_{X X} ; 211 \mathrm{~cm}^{-1}\right)=0.11$ are also calculated to be negligible to show strong polarization of the modes. Thus, the phonon assignment is performed relatively easily with minimal data collection for a diatomic molecule with tetragonal symmetry having a number of symmetry elements. 


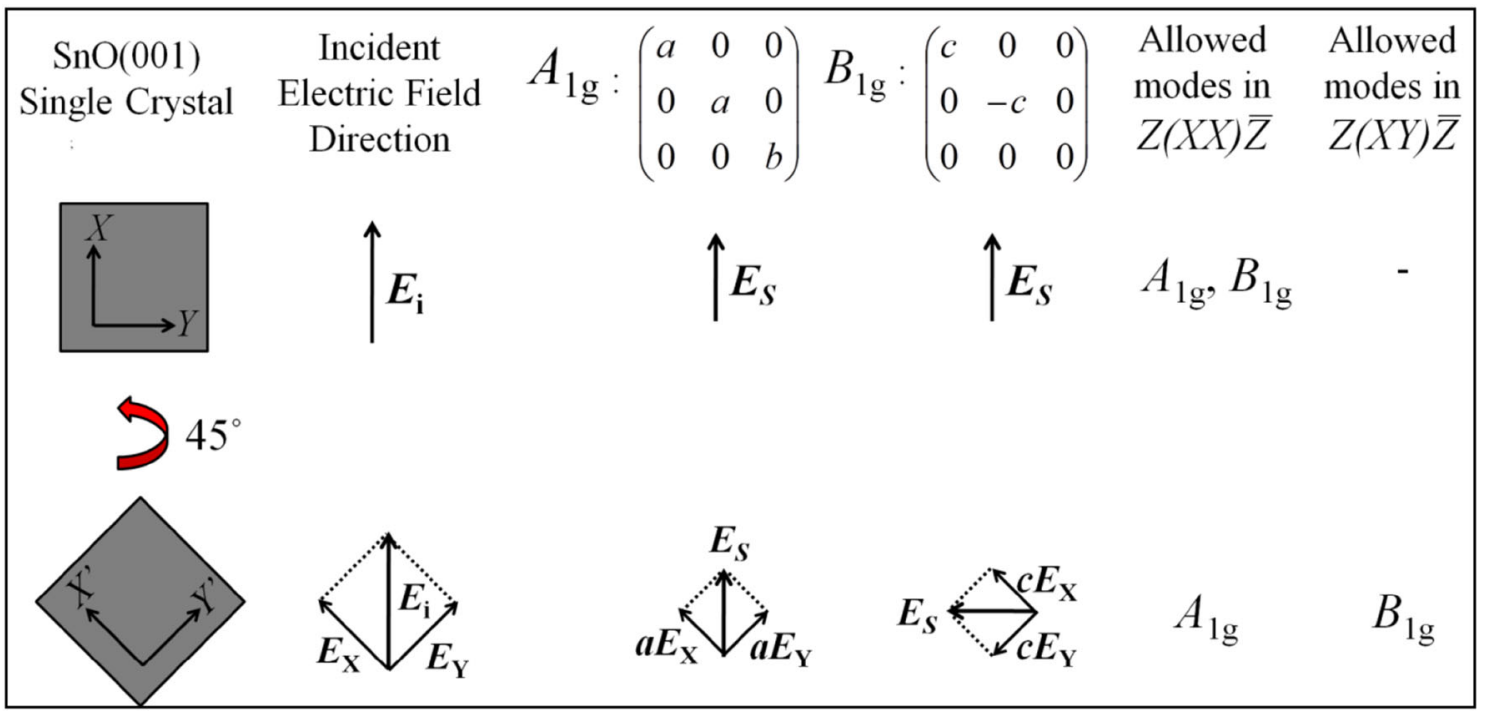

Figure 4. Direction of electric field vector with respect to incident electric field vector and prediction of allowed Raman modes in parallel and perpendicular polarization conditions for two different crystal orientations of the $\mathrm{SnO}(001)$ single crystal.

\section{Conclusions}

In conclusion, cubic- and cuboidal-shaped single crystals of $\mathrm{SnO}(001)$ were synthesized by the one-step hydrothermal method. Crystalline phase and orientation are identified using high resolution electron microscopy assisted structural and Raman spectroscopic studies. At the same time, polarized Raman studies of the sample only in two different crystal orientations: (i) $X$-axis parallel to incident electric field vector and (ii) oriented at an angle of $45^{\circ}$ around the $Z$-axis to incident electric field vector, confirming the crystal orientation. It also helps in solving the conflict in the assignment of the individual Raman modes uniquely, where $114 \mathrm{~cm}^{-1}$ mode is identified as $B_{1 \mathrm{~g}}$.

\section{Acknowledgement}

We thank V Sridharan of CMPD for his advice in preparing the sample and for his critical reading of the manuscript.

\section{References}

[1] Ogo Y, Hiramatsu H, Nomura K, Yanagi H, Kamiya T, Hirano M et al 2008 Appl. Phys. Lett. 9332113

[2] Liang L Y, Liu Z M, Cao H T and Pan X Q 2010 ACS Appl. Mater. Interfaces 21060

[3] Zhao Q and Bartsch R A 1995 J. Appl. Polym. Sci. 571465

[4] Han Z, Guo N, Li F, Zhang W, Zhao H and Qian Y 2001 Mater. Lett. 4899

[5] Pan X Q and Fu L 2001 J. Appl. Phys. 896048
[6] Uchiyama H and Imai H 2007 Cryst. Growth Des. 7841

[7] Ning J, Jiang T, Men K, Dai Q, Li D, Wei Y et al 2009 J. Phys. Chem. C 11314140

[8] Kumar B, Lee D H, Kim S H, Yang B, Meang S and Kim S W 2010 J. Phys. Chem. C 11411050

[9] Aurbach D, Nimberger A, Markovsky B, Levi E, Sominski E and Gedanken A 2002 Chem. Mater. 144155

[10] Dai Z R, Pan Z W and Wang Z L 2003 Adv. Funct. Mater. 139

[11] Orlandi M O and Leite E R 2006 J. Phys. Chem. B 1106621

[12] Geurts J, Rau S, Richter W and Schmitte F J 1984 Thin Solid Films 121217

[13] Wang X, Zhang F X, Loa I, Syassen K, Hanfland M and Mathis Y L 2004 Phys. Status Solidi B 2413168

[14] Luo H, Liang L Y, Cao H T, Liu Z M and Zhuge F 2012 ACS Appl. Mater. Interfaces 45673

[15] Liu Q, Liang L, Cao H, Luo H, Zhang H, Li J et al 2015 J. Mater. Chem. C 31077

[16] Iqbal M Z, Wang F, Din R U, Rafique M Y, Javed Q U A, Ullah A et al 2012 Mater. Lett. 7850

[17] Hota M K, Caraveo-Frescas J A, McLachlan M A and Alshareef H N 2014 Appl. Phys. Lett. 104152104

[18] Peltzer y Blanca E L, Svane A, Christensen N E, Rodriguez C O, Cappannini O M and Moreno M S 1993 Phys. Rev. B: Condens. Matter Mater. Phys. 4815712

[19] Koval S, Burriel R, Stachiotti M G, Castro M, Migoni R L, Moreno M S et al 1999 Phys. Rev. B: Condens. Matter Mater. Phys. 6014496

[20] Sornadurai D, Sridharan V, Ajikumar P K, Ravindran T R and Subramanian N 2017 AIP Conf. Proc. 1832140049

[21] Dai Z R, Pan Z W and Wang Z L 2002 J. Am. Chem. Soc. 124 8673

[22] Doh W H, Jeong W, Lee H, Park J and Park J Y 2016 Nanotechnology 27335603

[23] Kroumova E, Aroyo M I, Perez Mato J M, Kirov A, Capillas C, Ivantchev S et al 2003 Phase Transit. 76155 